\title{
THE HAND AS A SIGN-POST TO OCULAR DISEASE-V
}

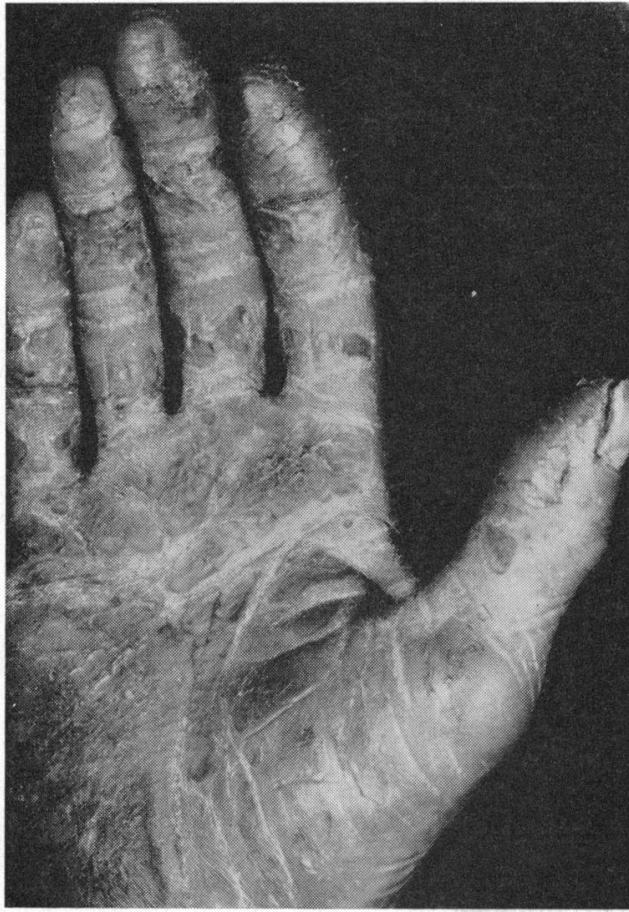

\section{Ichthyosis \\ Genodermatoses \\ This hereditary condition is characterized} by hyperkeratosis or hypertrophy of the horny layers of the skin which is dry and scaly and looks dirty (Fig. 1). Besides dominant, autosomal recessive, and sex-linked recessive forms, ichthyosis also occurs in the SjögrenLarsson syndrome (ichthyosis, spasticity, and oligophrenia) and in Refsum's syndrome, a disturbance of lipid metabolism (ichthyosis, atypical retinitis pigmentosa, polyneuritis, and deafness).

Blepharitis is common in ichthyosis and may cause a punctate keratitis, and, in the rare recessive ichthyosiform erythroderma, ectropion sometimes occurs. The characteristic corneal appearance, however, is a diffuse punctate change in the deeper layers of the stroma, while cataracts have also been reported.

FIG. 1.--Ichthyosis

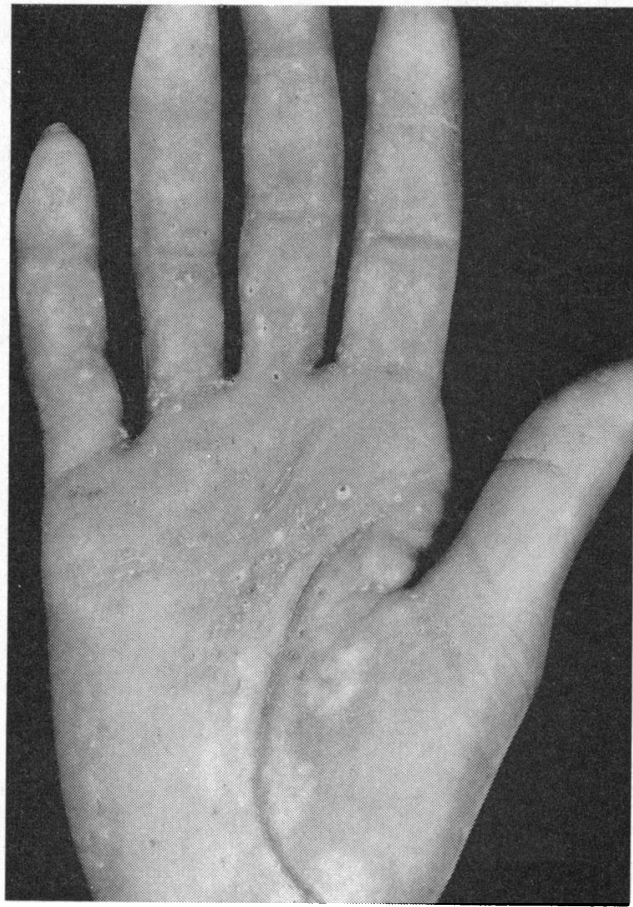

Tylosis (Keratosis palmaris et plantaris)

Tylosis appears within the first few years of life and is characterized by hyperkeratosis of the palms and soles, due to thickening of the horny layers of the epidermis (Fig. 2). The normal palmar creases are exaggerated and are often painful. The condition may have a dominant or a recessive mode of inheritance, the dominant form being occasionally associated with carcinoma of the oesophagus.

The typical ocular changes, which have been reported only in the recessive form, consist of lesions in the corneal epithelium which have a dendritic appearance.

Fig. 2.-Tylosis 


\section{Epidermolysis Bullosa}

This rare genetically-determined disease is either present at birth or appears early in life. Vesicles and blisters, frequently precipitated by minor trauma, occur in the skin and sometimes in mucous membranes. Scarring of these lesions occurs in the dystrophic form; it is not a feature of the more common simplex form. In dystrophic epidermolysis bullosa, the nails and fingers are often atrophic (Fig. 3) and an acquired syndactyly may result.

Vesicles may occur in the conjunctiva in the dystrophic form and may result in the formation of symblepharon; if they involve the cornea, ulceration is characteristically followed by subepithelial infiltration of vascularized scar tissue, though perforation of the cornea has been reported.

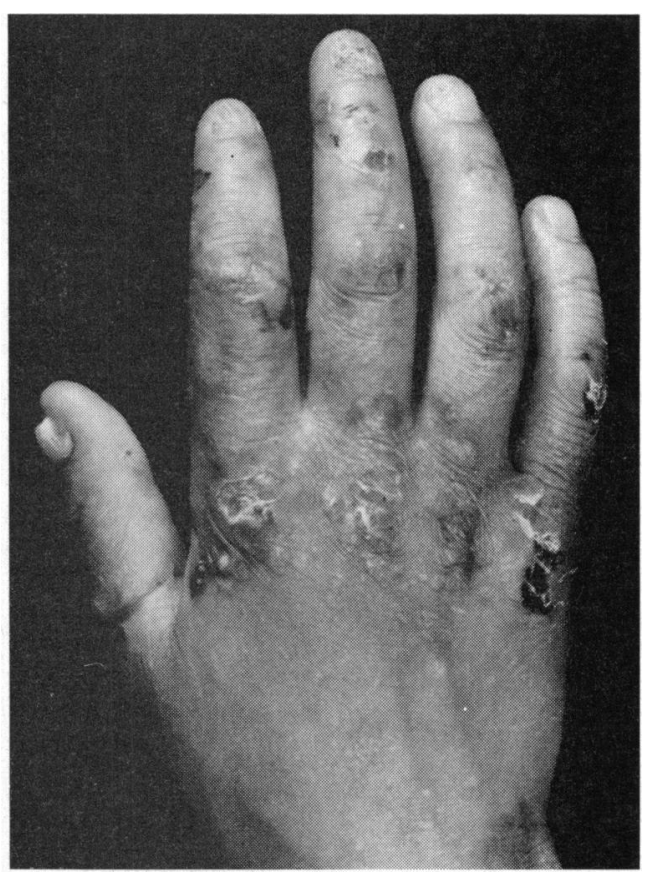

FIG. 3.-Epidermolysis bullosa

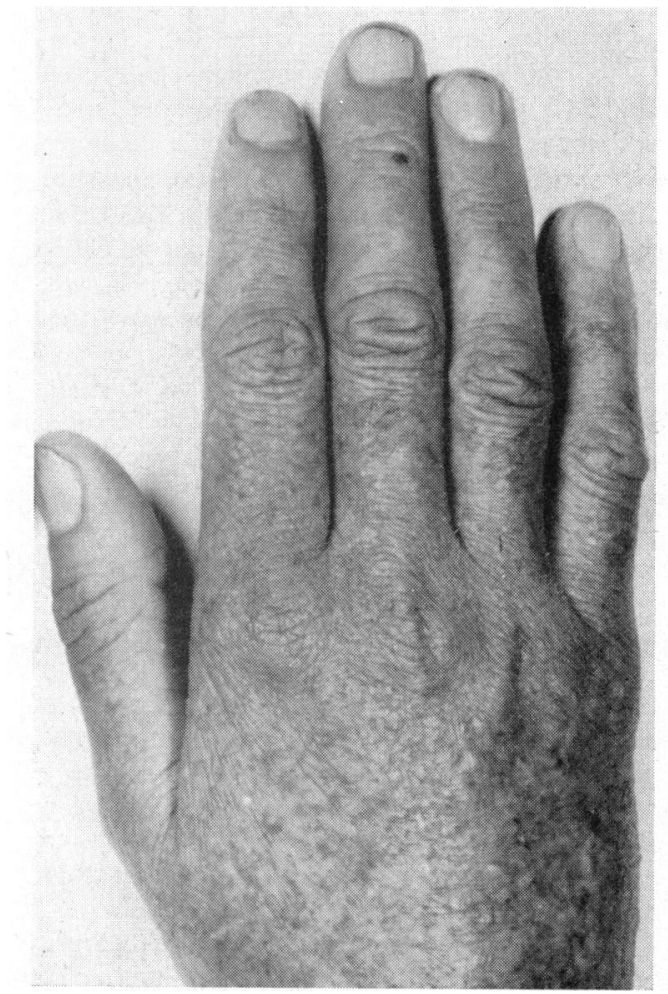

Fig. 4.-Xeroderma pigmentosum

\section{Illustrations}

St John's Hospital for Diseases of THE SKIN,

BarRie JAY AND M. D. SANDERS, MOORFIELDS EYE HOSPITAL, LONDON, W.C.1.

This rare condition is characterized by freckles, telangiectases, atrophy of the skin (Fig. 4), and the formation of warty tumours on exposed areas of skin. These tumours frequently undergo malignant change into carcinomata (basal cell and squamous cell) or face and hands.

Photophobia is often the presenting symptom, and erythema of the skin of the eyelids and conjunctival hyperaemia may be ited by exposure to sunlight.

Corneal infiltrates are common and limba changes. Areas of extreme atrophy of the iris have been reported.

\section{LONDON, W.C.2.}

